\title{
BMJ Open The prevalence, impact and cost of chronic non-cancer pain in Irish primary schoolchildren (PRIME-C): protocol for a longitudinal school-based survey
}

\author{
Siobhán O’Higgins, ${ }^{1}$ Edel Doherty, ${ }^{2}$ Saoirse NicGabhainn, ${ }^{3}$ Andrew Murphy, ${ }^{4}$ \\ Michael Hogan, ${ }^{5}$ Ciaran O'Neill, ${ }^{2}$ Brian E McGuire ${ }^{1}$
}

To cite: O'Higgins S, Doherty E, NicGabhainn S, et al. The prevalence, impact and cost of chronic noncancer pain in Irish primary schoolchildren (PRIME-C): protocol for a longitudinal school-based survey. BMJ Open 2015;5:e007426. doi:10.1136/bmjopen-2014007426

- Prepublication history for this paper is available online. To view these files please visit the journal online (http://dx.doi.org/10.1136/ bmjopen-2014-007426).

Received 11 December 2014 Accepted 23 December 2014

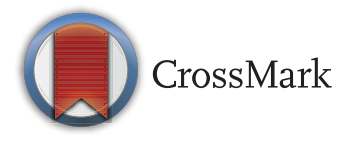

For numbered affiliations see end of article.

Correspondence to Dr Siobhán O'Higgins; siobhan.ohiggins@nuigalway

\section{ABSTRACT}

Introduction: Previous research has indicated that pain influences children's daily lives, resulting in absence from school, sleep problems, poor school performance and problems with social activities. Our study aims to characterise the nature, extent, impact and cost of chronic pain among Irish children.

Methods and analysis: Using cluster-systematic random sampling, primary schools will be invited to participate and 6000 5-12-year-olds asked to complete questionnaires in school classrooms, at time points 1 year apart. Questionnaires will use internationally valid psychometric measures to assess a range of quality of life factors and chronic pain indicators among children, with corresponding parental/primary caregiver questions, which will be completed at home. Data will also be gathered on the cost of chronic pain. Space will be given for comments on how pain impacts on participants' lives and possible coping mechanisms.

Ethics and dissemination: Ethical approval has been granted by the Research Ethics Committee, National University of Ireland, Galway.

Dissemination of results will be via journal articles and conference presentations on the various aspects of the study (ie, prevalence, impact and economic cost of chronic pain among 5-12-yearolds living in Ireland).

\section{PREVIOUS RESEARCH}

In most cases, the typical transitory aches and pains that children suffer following injury or medical procedures do not upset daily activities or disrupt their psychosocial development. ${ }^{1}$ Chronic pain, on the other hand, is a persistent health problem that can, on a regular basis, upset participation in social and physical activities, and lead to frequent school absences. $^{2-5}$ Pain that persists

\section{Strengths and limitations of this study}

- Despite extant research and developments in paediatric pain care, many children and adolescents still suffer because of inadequate pain treatments.

- Data are necessary to inform policymakers and funders as to the need for tailored resources and interventions for children with chronic pain.

- This is the first study of chronic pain prevalence, impact and cost among primary schoolchildren living in Ireland.

- The piloting stage of this study will ensure that valid and reliable measures of pain intensity and impact are developed suitable for children.

- This is a prevalence study, so it will not have the capacity to explore, in depth, how paediatric chronic pain impacts on family life. A qualitative study examining these issues, once prevalence and costs are established, could be warranted.

or recurs for 3 months or longer is considered chronic pain. ${ }^{6}$ Pain-related limitations can interfere with a child's sense of competence, quality of life and effective functioning in academic, vocational and social areas of life, as these require regular participation in activities such as school and social interactions. ${ }^{17}$

Previous studies on chronic pain among children in Europe have established that it is a common public health issue. A study of children aged 8-18 years in Norway found that children with chronic pain reported reduced social life, increased sleep disturbance, fewer hobbies and more absences from school. A recent meta-analysis revealed a prevalence of paediatric chronic pain between $11 \%$ and $38 \% .^{8}$ Palermo et al noted that children with chronic pain are at greater 
risk of anxiety and depression, and increased risk of ongoing pain in adulthood. Adolescents with chronic pain are at higher risk of impairment in their friendships, are less socially developed, and at higher risk of suicidal ideation and attempting suicide. ${ }^{9} 10$

Prevalence studies may be either registry based or use patient self-report. Self-rated health is based on an individual's perception and evaluation of his or her health. ${ }^{11}$ In this study, we will ask children about their health-related quality of life (HRQoL) and questions that pertain to chronic pain; we will also ask parents/ primary caregivers about pain and other health problems in their children.

Our earlier study (the prevalence, impact and cost of chronic non-cancer pain, PRIME) established the prevalence of chronic pain among adults living in Ireland as $35.5 \%{ }^{12}$ and identified the cost as $€ 5665$ per person, per year, or $2.86 \%$ of GDP. ${ }^{13}$ Currently, there is little information available on the extent of chronic pain and painrelated difficulties in Irish schoolchildren. There are two other ongoing population studies on Irish childhood, the Health Behaviours of School-Aged Children $(\mathrm{HBSC}))^{14}$ and the longitudinal Growing Up in Ireland study, (http://www.growingup.ie), both providing important information on the health profile of Irish children. However, these studies do not evaluate the nature, extent and impact of chronic pain.

The prevalence impact and cost of chronic noncancer pain among Irish primary schoolchildren (PRIME-C) study described here will generate novel and important data on the experience of chronic pain among children aged 5-12 years living in the Republic of Ireland.

\section{RESEARCH AIM}

The PRIME-C study aims to identify the prevalence and impact of chronic pain among primary schoolchildren aged 5-12 year and their families living in Ireland, and the economic cost of chronic pain in children. Time and attention will also be given to facilitate children to tell the story of their pain.

\section{METHOD/DESIGN}

A longitudinal school-based survey of children in two age groupings, 5-8 and 9-12 years, collected on at least two time points 1 year apart. A parallel study will be conducted with parents/primary caregivers of the children, at the same time points, with questionnaires to be completed at home. Data collection began in 2012 and will continue at least until summer 2015.

\section{Recruitment and eligibility}

\section{Sample size and power calculation}

Most previous studies ${ }^{8}$ suggest that $15-25 \%$ of children have chronic pain. To ensure an adequate sample, we will adopt a conservative approach and the lower figure will be used to calculate the sample size. Assuming that
$15 \%$ of our sample may exhibit chronic pain, this suggests that for a total of 1000 responses, $95 \%$ confidence limits will be $12.9 \%$ and $17.4 \%$ (StataCorp 2005, Stata statistical software: Release V.9. College Station, Texas, USA: StataCorp). With an estimated response rate of $40 \%$, based on the original PRIME study, ${ }^{11}$ this suggests a total sampling frame of 2500 .

To adjust for the cluster sampling frame by school, we have been unable to identify an appropriate intracluster correlation (ICC). We have therefore calculated the design effect using the median ICC $(0.010) .{ }^{15}$ Based on an average recruitment of 100 students per school, the design effect is 1.99 and the total required sample will be 4975 .

As we intend to have follow-ups at 1-year intervals and our previous experience with the PRIME study was of a $60 \%$ response rate at time $2(60 \%$ of those who had responded at time 1), we wish to ensure an adequate 'pool' of respondents at time 1. In addition, as the nature, prevalence and impact of chronic pain may also vary at different stages of childhood, we wish to obtain a sufficient sample of respondents from each age cohort (5-8 and 9-12 years). For this reason, we intend to expand our sampling frame to a maximum of 6000 (3000 per age cohort).

\section{Sampling}

The study will use cluster-systematic random sampling: data stratified by regions and clustered by schools. Stratification by geographical regions will reduce any bias due to different access to health services and variance in disease patterns. Schools will be randomly selected within each region. The regional stratification will be by the demarcation of eight regions as allocated by the Department of Education and Skills of Ireland. The sampling frame will be provided by the Department (ie, their list of primary schools). This list will be divided by region, and the number of students to be sampled from each will be determined by the distribution of children aged 5-12 years in each region, according to the Irish Census, 2011. Each school from within each area list will receive a random number generated by Excel Software. The schools will then be sorted by their random number, in order to diminish any systematic order that might exist in the list received from the Department of Education and Skills.

\section{Recruitment}

Invitations to participate will be sent to school principals. Once the principals have accepted the invitation to participate, researchers will visit every class group twice. The first visit will be to introduce the study, stressing the important role of each student within it and asking the students to take home a letter for their parents or primary caregiver. They will be asked to return the form with their parents/primary caregiver's consent for their child to participate. The second visit will be to conduct data collection, at which time each child will also be 
asked for his or her written assent to participate. Only students who have given assent and for whom there is parental consent will be asked to participate.

\section{Measures}

Several systematic reviews of paediatric pain measures have been published recently as knowledge increases about the negative effects of chronic pain on children. ${ }^{16}$ Such reviews formed the basis for selecting the most appropriate measures for the age groups under scrutiny. The items selected for inclusion in our survey instruments are standardised, reliable and valid psychometric measures used internationally to assess a range of quality of life factors and chronic pain indicators among children. When working with younger children or children with educational difficulties, the questionnaire will be delivered orally by the research team. Our selected measures all have corresponding parent/primary caregiver questions.

Demographic, KINDL ${ }^{\mathrm{R}}$ and Kiddy $\mathrm{KINDL}^{\mathrm{R}}$ quality of life data will be included for comparison between those who do and do not have chronic pain. Items will be adapted from the following:

1. $\mathrm{KINDL}^{\mathrm{R}} ;{ }^{17}$ this is a generic measure of children's HRQoL. It consists of 24 Likert-scaled items associated with six dimensions: physical well-being, emotional well-being, self-esteem, family, friends and everyday functioning (school or nursery school/kindergarten). It has a 5-point frequency scale $(1=$ never, $2=$ almost never, $3=$ sometimes, $4=$ often, $5=$ all the time), where scores across the six dimensions can be totalled to give an overall score out of 100. Higher scores then reflect a better quality of life.

Kiddy $\mathrm{KINDL}^{\mathrm{R}}$, ${ }^{17}$ a shorter version of $\mathrm{KINDL}^{\mathrm{R}}$, designed for use with younger children, has half the number of items within the six dimensions and a 3-point frequency scale ( $1=$ never, $2=$ sometimes, $3=$ all the time), and will be used for the 5-8-year-olds. The subscales of these six dimensions can be combined to produce a total score. All the children in the survey, whether or not they have pain, will complete the $\mathrm{KINDL}^{\mathrm{R}}$ or Kiddy $\mathrm{KINDL}^{\mathrm{R}}$.

2. Case screening questions will determine if the child experiences chronic pain, defined by the International Association for the Study of Pain (IASP) ${ }^{18}$ as persistent pain that lasts at least 3 months.

3. Those answering 'yes' to the screening questions will complete additional measures on the history and nature of their pain, site(s) of pain, time since onset and any known cause for the pain, such as an accident or injury, and any medical diagnoses. The Varni-Thompson Paediatric Pain ${ }^{19}$ measure will be used to assess intensity and location of pain. Pain intensity is measured on two $10 \mathrm{~cm}$ lines by placing 'a mark on the line between the faces $(1$ sad and 1 happy) that best shows how much pain you are feeling now' and 'the worst pain you had this week'. The children will be asked to mark on an outline of a body (front and back views) the location(s) of their pain. By using one of three colours, pain intensity will also be indicated as 'a little pain', 'some pain' or 'a lot of pain' in each of the areas identified as being painful.

4. An additional $\mathrm{KINDL}^{\mathrm{R}}$ subscale entitled 'Disease' will be used to measure the child's perceptions of quality of life with respect to his or her pain; with 'pain' substituted for 'illness' in the six items. Scoring of this subscale is as described above in relation to the full KINDL $^{\mathrm{R}}$.

5. The Bath Adolescent Pain Questionnaire (BAPQ) ${ }^{20} 21$ to measure self-reported functional disability associated with pain using seven domains: social development, social, physical and family functioning, depression, general and pain-specific anxiety. It is envisaged that those aged 5-8 years, who self-identify as having chronic pain, will similarly fill in all dimensions (on an adapted 3-point frequency scale, based on the age-appropriate scale of the Kiddy $\mathrm{KINDL}^{\mathrm{R}}$ ). Here, higher scoring will indicate greater impairment.

The parental/primary caregiver versions of $\mathrm{KINDL}^{\mathrm{R}}$ and BAPQ include six dimensions and 5-point frequency scales.

The parents will also be queried on their age range, gender, ethnicity, education, income levels, current employment and marital status. These will help identify the socioeconomic profile of families. They will also be asked about their own health including whether they suffered from chronic pain or any other illness; they will, in addition, be asked their children's age, gender and presence of chronic illness. A section is included in the parental questionnaire to identify the healthcare and non-healthcare costs of children's chronic pain.

\section{Economic measures}

The parental/primary caregiver survey contains an additional section on healthcare services usage and financial costs of the child's chronic pain. In the case of healthcare service use, questions will be asked to all parents regarding their children's use of primary healthcare services, hospital services and other community healthcare services, such as psychology and physiotherapy services. Unit cost estimates will be used to translate healthcare service utilisation into a cost figure for each healthcare service. In addition, out of pocket expenses such as costs of travelling to and waiting at healthcare clinics will be recorded. For children with chronic pain, parents are asked to provide an overview of medication use and costs to treat the pain, and any other costs such as those associated with the provision of any special equipment. Information will also be recorded on formal and informal caregiving to children with chronic pain.

\section{Pilot study}

We will conduct a pilot study to ensure that the child questionnaires are age-appropriate, acceptable, understandable and adopt a user-friendly format. A pilot study 
will also be conducted with parents; their questionnaires will have additional items to quantify the direct economic burden associated with their children's chronic pain. The questionnaires will then be reviewed and assessed by the research team to ensure face validity is retained.

A full pilot study will be conducted within one local school to evaluate the logistic feasibility of the full-scale study, focusing on ironing out any problems that may arise with data collection and protocol adherence.

\section{Data collection}

Working with primary schoolchildren, data collection is envisaged to take a full school day for each school. In larger schools, one class group from each year will be selected randomly, where possible, from information provided by the schools (from previous experience, this may not always be possible, as principals tend to select class groups based on timetable restrictions on the day of data collection). Younger age groups and those with educational disabilities will be assisted by the research team. Those answering 'no' to the screening questions will be asked to draw a picture in their booklets; hence all participants will finish their questionnaire at about the same time. For those children whose parents give consent but are absent on the day, questionnaires will be left with class teachers. Support within each school will be vital to ensure parental consent forms and surveys are requested for and returned. Thus time will be spent explaining the objectives and importance of the survey to all teachers during the breaks in class. Parents' surveys will be returned to the school, and then send large 'freepost' envelopes full of surveys back to the Centre for Pain Research, National University of Ireland, Galway.

\section{Analysis}

The associations between the constructs (sociodemographics, family structures, child gender, age, perceptions of HRQoL, child and parental chronic pain reports, perceptions of impact, healthcare use and economic costs) will be assessed using multivariate regression, hierarchical linear regression and mediation analyses (ie, bootstrap methodology). To determine whether significant differences in household and parental characteristics exist between children with and without pain, a series of student $t$ tests and $\chi^{2}$ tests will be undertaken. Binary logistic regression models will further estimate with ORs presented to explore what factors are significantly associated with a child reporting chronic pain in multivariate analyses. The factors will include child, parental and household characteristics. Multivariate regression analysis will also be undertaken to explore factors associated with functional disability, for those children who report having chronic pain, based on the BAPQ questionnaire. Univariate and multivariate analysis will also be conducted to estimate the incremental costs of chronic pain in children.

\section{ETHICAL CONSIDERATIONS}

Importance is placed on the research process respecting the rights, well-being and dignity of those involved or affected. $^{22}$ Following written parental consent being obtained, the child participants will also be asked for their written assent. It will be stressed that all participants are free to withdraw at any time during the research process, without any negative impact on them. All completed surveys will be anonymised by number and kept in secure storage. The data will be entered into SPSS via codes for each school and each participant's family, and raw data will be stored in the data storage facility in the School of Psychology for 5 years as required by the Research Ethics Committee. Only members of the researcher team will have access to the codes, stored in a separate file.

Although there are no apparent risks in administering the survey, either physical or psychological, provision has been made to respond to those children who are identified as being distressed.

The lead researcher's contact details will be available to all participants should they wish, at any point, to discuss concerns about the study.

\section{Author affiliations}

${ }^{1}$ Centre for Pain Research, School of Psychology, National University of Ireland, Galway, Ireland

${ }^{2}$ Department of Economics, National University of Ireland, Galway, Ireland ${ }^{3}$ Health Promotion Research Centre, National University of Ireland, Galway, Ireland

${ }^{4}$ Discipline of General Practice, National University of Ireland, Galway, Ireland

${ }^{5}$ School of Psychology, National University of Ireland, Galway, Ireland

Contributors SO contributed in the design of the study and drafted the manuscript. BEM oversaw the project as principal investigator, and reviewed and edited the manuscript. SN and $\mathrm{MH}$ reviewed and edited the manuscript. ED and $\mathrm{CO}$ designed the health economic aspects of the study and reviewed the manuscript. AM contributed in the design of the study.

Funding This research is funded by the Health Research Board of Ireland Interdisciplinary Capacity Enhancement Award (Reference: ICE/2011/19).

Competing interests None declared.

Ethics approval Research Ethics Committee, National University of Ireland, Galway.

Provenance and peer review Not commissioned; peer reviewed for ethical and funding approval prior to submission.

Data sharing statement Dissemination of results will be via journal articles and conference presentations on the various aspects of the study (ie, prevalence, impact and economic cost of chronic pain among 5-12-year-olds living in Ireland).

Open Access This is an Open Access article distributed in accordance with the Creative Commons Attribution Non Commercial (CC BY-NC 4.0) license, which permits others to distribute, remix, adapt, build upon this work noncommercially, and license their derivative works on different terms, provided the original work is properly cited and the use is non-commercial. See: http:// creativecommons.org/licenses/by-nc/4.0/

\section{REFERENCES}

1. Carter BD, Threlkeld BM. Psychosocial perspectives in the treatment of pediatric chronic pain. Pediatr Rheumatol Online J 2012;10:15.

2. Claar RL, Walker LS, Smith CA. Functional disability in adolescents and young adults with symptoms of irritable bowel syndrome: the 
role of academic, social, and athletic competence. J Pediatr Psychol 1999;24:271-80.

3. Hainsworth KR, Davies WH, Khan KA, et al. Development and preliminary validation of the child activity limitations questionnaire: flexible and efficient assessment of pain-related functional disability. J Pain 2007;8:746-52.

4. Palermo $T$, Wilson A. Peters $M$, et al. Randomized controlled trial of an internet-delivered family cognitive-behavioral therapy intervention for children and adolescents with chronic pain. Pain 2009;146:205-13.

5. Dick BD, Pillai Riddell R. Cognitive and school functioning in children and adolescents with chronic pain: a critical review. Pain Res Manag 2010;15:238-44.

6. Merskey H, Bogduk N. Classification of chronic pain, IASP task force on taxonomy. 2nd edn. Seattle: IASP Press, 1994.

7. Walters AS, Williamson GM. The role of activity restriction in the association between pain and depression: a study of pediatric patients with chronic pain. Child Health Care 1999;28:33.

8. King S, Chambers CT, Huguet A, et al. The epidemiology of chronic pain in children and adolescents revisited: a systematic review. Pain 2011;152:2729-38.

9. Forgeron PA, McGrath P, Stevens B, et al. Social information processing in adolescents with chronic pain: my friends don't really understand me. Pain 2011;152:2773-80.

10. Eccleston C, Wastell S, Crombez G, et al. Adolescent social development and chronic pain. Eur J Pain 2008;12:765-74.

11. Bjorner JB, Kristensen TS, Orth-Gomér K, et al. Self-rated health. $A$ useful concept in research, prevention and clinical medicine. Stockholm: Swedish Council for Planning and Coordination of Research, 1996.

12. Raftery MN, Sarma K, Murphy AW, et al. Chronic pain in the Republic of Ireland-community prevalence, psychosocial profile and predictors of pain-related disability: results from the Prevalence,
Impact and Cost of Chronic Pain (PRIME) study, part 1. Pain 2011;152:1096-103.

13. Raftery MN, Ryan P, Normand C, et al. The economic cost of chronic noncancer pain in Ireland: results from the PRIME study, part 2. J Pain 2012;13:139-45.

14. Kelly C, Gavin A, Molcho M, et al. The Irish health behaviours in school-aged children (HBSC) study 2010. Dublin: Department of Health, 2012.

15. Adams G, Gulliford M, Ukoumunne O, et al. Patterns of intra-cluster correlation from primary care research to inform study design and analysis. J Clin Epidemiol 2004;57:785-94.

16. Huguet A, Stinson JN, McGrath PJ. Measurement of self-reported pain intensity in children and adolescents. J Psychosom Res 2010;68:329-36.

17. Ravens-Sieberer U, Bullinger $M$. The revised KINDL-R: final results on reliability, validity and responsiveness of a modular HRQOL instrument for children and adolescents. Qual Life Res 2001;10:199.

18. International Association for the Study of Pain. Classification of chronic pain. Descriptions of chronic pain syndromes and definitions of pain terms. Pain 1986(Suppl 3):S1-226.

19. Varni JW, Seid M, Kurtin PS. PedsQLTM 4.0: reliability and validity of the Pediatric Quality of Life Inventory ${ }^{\mathrm{TM}}$ version 4.0 generic core scales in healthy and patient populations. Med Care 2001;39:800-12.

20. http://www.bath.ac.uk/pain (accessed 2013)

21. Eccleston C, Jordan A, McCracken LM, et al. The Bath Adolescent Pain Questionnaire (BAPQ): development and preliminary psychometric evaluation of an instrument to assess the impact of chronic pain on adolescents. Pain 2005;118:263-70.

22. Kilkelly U, Donnelly M. The child's right to be heard in the healthcare setting: perspectives of children, parents and health professionals. Office of the Minister for Children and Youth Affairs, 2006. 